\title{
Comparison of weighted-density-functional theories for inhomogeneous liquids
}

\author{
D. M. Kroll and Brian B. Laird \\ Institut für Festkörperforschung des Forschungszentrums Jülich, Postfach 1913, 5170 Jülich, West Germany
}

(Received 22 May 1990)

\begin{abstract}
Three recent weighted-density-functional (WDF) theories are critically examined in terms of their ability to describe correctly the structure of a hard-sphere fluid at a hard wall. A new derivation of the Curtin-Ashcroft WDF theory is given that clarifies the basic approximations behind this formulation and brings out the close relationship between their work and the WDF theories of MeisterKroll and Groot-van der Eerden. The condition that the second functional derivative of the approximate Helmholtz free-energy functional yields the correct two-particle direct-correlation function in the homogeneous limit is found to be of crucial importance in determining good liquid structures.
\end{abstract}

\section{INTRODUCTION}

One of the most demanding tests of theories of inhomogeneous fluids, i.e., fluids whose density varies significantly over molecular dimensions, is the prediction of the fluid structure near solid surfaces. Particular problems arise due to the necessity of correctly describing multiphase coexistence, while simultaneously incorporating a sufficiently detailed treatment of the rapid variation of the density profiles that can arise in interfacial regions.

Density-functional methods have proven to be one of the most successful approaches for studying both the structure of inhomogeneous classical liquids and freezing. Most of these theories use rather similar physical concepts. For most simple liquids, it is generally assumed that the intermolecular interaction can be divided into a short-range repulsive part $u_{r}(r)$, which determines the structure of the liquid, and a long-range attractive tail $u_{a}(r){ }^{1}$ For most applications it is sufficient to treat $u_{a}(r)$ in the mean-field approximation. The real test of the various density-functional theories therefore lies in their ability to reproduce the short-range structure induced by $u_{r}(r)$. The standard test case is the structure of a hardsphere liquid at a hard wall. In this paper we compare the quality of the density profiles predicted for this case by three recent weighted-density-functional theories. ${ }^{2-4}$ These theories (described in Sec. II) are among the most sophisticated currently available so that our results give some indication of the accuracy that is currently attainable. More important, the comparison yields valuable information regarding the quality of the various approximations that have gone into the formulation of these theories and should be of use in future attempts to develop improved approximations.

\section{WEIGHTED-DENSITY FUNCTIONALS}

The basic assumption of all density-functional theories is that the thermodynamic potential of an inhomogeneous system can be approximated using known structural and thermodynamic properties of the corresponding homogeneous fluid. The most successful density func- tionals are based on the so-called weighted-density approximation (WDA). In this approach, an approximate free-energy functional is constructed in such a way that the free-energy density of an inhomogeneous liquid at a given point is interpreted as that of a homogeneous system taken at an auxiliary local average (coarse-grained) density.

Several early attempts to implement this idea were rather successful. ${ }^{5-10}$ In particular, these schemes succeeded in locating a first-order freezing transition ${ }^{7,8}$ for simple liquids in qualitative agreement with simulation studies and gave reasonably good results for the structure of a liquid in contact with a wall. ${ }^{5,6,9,10}$ However, these theories were of a rather ad hoc nature in that they did not result from well-defined approximations on the exact functional.

Subsequent developments can be summarized as follows: The Helmholtz free energy $\mathscr{F}[\rho]$, which is a unique functional of the one-particle density $\rho(\mathbf{r})$, has the form

$$
\mathscr{F}[\rho]=\mathscr{F}_{\text {id }}[\rho]+\mathscr{F}_{\text {ex }}[\rho],
$$

where

$$
\mathcal{F}_{i d}[\rho]=\beta^{-1} \int d \mathbf{r} \rho(\mathbf{r})\left\{\ln \left[\Lambda^{3} \rho(\mathbf{r})\right]-1\right\}
$$

is the ideal-gas contribution and

$$
\mathcal{F}_{\mathrm{ex}}[\rho]=-\beta^{-1} \int d \mathbf{r} \rho(\mathbf{r}) \int_{0}^{1} d \lambda c^{(1)}(\mathbf{r} ;[\lambda \rho]),
$$

where $\quad \beta^{-1} c^{(1)}(\mathbf{r} ;[\rho])=-\delta \mathcal{F}_{\mathrm{ex}} / \delta \rho(\mathbf{r}) . \quad$ Expanding $c^{(1)}(\mathbf{r} ;[\rho])$ about a homogeneous liquid with the coarsegrained density $\rho_{0}(\mathbf{r})$, one has

$$
\begin{array}{r}
c^{(1)}(\mathbf{r} ;[\rho])=c^{(1)}\left(\rho_{0}(\mathbf{r})\right)+\int d \mathbf{r}^{\prime} \int_{0}^{1} d \lambda^{\prime} c^{(2)}\left(\mathbf{r}, \mathbf{r}^{\prime} ;\left[\rho \lambda^{\prime}\right]\right) \\
\times\left[\rho\left(\mathbf{r}^{\prime}\right)-\rho_{0}(\mathbf{r})\right]
\end{array}
$$

where $c^{(2)}\left(\mathbf{r}, \mathbf{r}^{\prime} ;[\rho]\right)$ is the two-particle direct-correlation functional of the inhomogeneous liquid, $c^{(1)}\left(\rho_{0}(\mathbf{r})\right)$ is the one-particle direct-correlation function of a homogeneous liquid at density $\rho_{0}(\mathbf{r})$, and $\rho_{\lambda^{\prime}}\left(\mathbf{r}^{\prime}\right)=\rho_{0}(\mathbf{r})$ $+\lambda^{\prime}\left[\rho\left(\mathbf{r}^{\prime}\right)-\rho_{0}(\mathbf{r})\right]$. Finally, substituting this result in (3) 
yields

$$
\begin{aligned}
\mathcal{F}_{\mathrm{ex}}[\rho]=\int & d \mathbf{r} \rho(\mathbf{r}) f_{0}\left(\rho_{0}(\mathbf{r})\right)-\beta^{-1} \\
& \times \int d \mathbf{r} \int d \mathbf{r}^{\prime} \rho(\mathbf{r}) \mathcal{C}\left(\mathbf{r}, \mathbf{r}^{\prime} ;[\rho]\right)\left[\rho\left(\mathbf{r}^{\prime}\right)-\rho_{0}(\mathbf{r})\right],
\end{aligned}
$$

where $f_{0}\left(\rho_{0}\right)$ is the excess Helmholtz free energy per particle of the homogeneous liquid at density $\rho_{0}$ and

$$
\mathcal{C}\left(\mathbf{r}, \mathbf{r}^{\prime} ;[\rho]\right)=\int_{0}^{1} d \lambda \lambda \int_{0}^{1} d \lambda^{\prime} c^{(2)}\left(\mathbf{r}, \mathbf{r}^{\prime} ;\left[\lambda \rho_{\lambda^{\prime}}\right]\right) .
$$

This expression for $\mathscr{F}_{\text {ex }}[\rho]$ is exact. Because little is known about the functional $c^{(2)}\left(\mathbf{r}, \mathbf{r}^{\prime} ;[\rho]\right)$ for inhomogeneous systems, practical applications involve utilizing knowledge of the properties of the homogeneous system. Since no detailed information exists for the three-particle and higher direct-correlation functions, even for homogeneous systems, the approach followed by Meister and Kroll (MK) in Ref. 2 was to expand the right-hand side of (5) and truncate after the bulk $c^{(2)}$ term. As a result, one obtains

$$
\begin{aligned}
\mathcal{C}\left(\mathbf{r}, \mathbf{r}^{\prime} ;[\rho]\right) & \approx S\left(\left|\mathbf{r}-\mathbf{r}^{\prime}\right| ; \rho_{0}(\mathbf{r})\right) \\
& =\int_{0}^{1} \lambda d \lambda c^{(2)}\left(\left|\mathbf{r}-\mathbf{r}^{\prime}\right| ; \lambda \rho_{0}(\mathbf{r})\right) .
\end{aligned}
$$

The terms neglected in (6) are proportional to $\lambda$ integrals of higher-order direct-correlation functions. In the approach of $\mathrm{MK}$, the coarse-graining procedure is then defined by extremizing the resulting expression for $\mathcal{F}_{\text {ex }}\left[\rho, \rho_{0}\right]$ with respect to $\rho_{0}(\mathbf{r})$, the motivation being to minimize the dependence of $\mathcal{F}_{\text {ex }}$ on $\rho_{0}$. As a result, one finds that

$$
\rho_{0}(\mathbf{r})=\int d \mathbf{r}^{\prime} w\left(\left|\mathbf{r}-\mathbf{r}^{\prime}\right| ; \rho_{0}(\mathbf{r})\right) \rho\left(\mathbf{r}^{\prime}\right),
$$

where the weight function $w\left(\left|\mathbf{r}-\mathbf{r}^{\prime}\right| ; \rho_{0}(\mathbf{r})\right)$ $=Q^{\prime}\left(\left|\mathbf{r}-\mathbf{r}^{\prime}\right| ; \rho_{0}(\mathbf{r})\right) / S_{0}^{\prime}\left(\rho_{0}(\mathbf{r})\right)$, with $S^{\prime}(r ; \rho)=\partial S / \partial \rho$ and $\mathcal{S}_{0}^{\prime}(\rho)=\int d \mathbf{r}{S^{\prime}}^{\prime}(r, \rho)$.

An attractive feature of this approach is that the coarse-graining procedure follows quite naturally from the requirement that $\delta \mathcal{F}_{\mathrm{ex}} / \delta \rho_{0}(\mathbf{r})=0$. Unfortunately, although this approximation is consistent with the compressibility equation of state and various sum rules, it does not satisfy the relation

$$
\left.\beta \frac{\delta^{2} \mathcal{F}_{\mathrm{ex}}}{\delta \rho(\mathbf{r}) \delta \rho\left(\mathbf{r}^{\prime}\right)}\right|_{\rho(\mathbf{r})=\rho}=-c^{(2)}\left(\left|\mathbf{r}-\mathbf{r}^{\prime}\right| ; \rho\right)
$$

in the homogeneous bulk phase.

Subsequently, Groot and van der $\operatorname{Eerden}^{3}(\mathrm{GE})$ extended this model to incorporate condition (8). They start by approximating $\mathcal{C}\left(\mathbf{r}, \mathbf{r}^{\prime} ;[\rho]\right)$ in $(5)$ by an effective two-point function of a homogeneous liquid at the local density $\rho_{0}(\mathbf{r})$

$$
\mathcal{C}\left(\mathbf{r}, \mathbf{r}^{\prime} ;[\rho]\right) \approx S\left(\left|\mathbf{r}-\mathbf{r}^{\prime}\right| ; \rho_{0}(\mathbf{r})\right) .
$$

The condition $\delta \mathcal{F}_{\mathrm{ex}} / \delta \rho_{0}(\mathbf{r})=0$, applied in the homogeneous state $\rho(\mathbf{r})=\rho_{0}(\mathbf{r})=\rho$, implies then that $S_{0}(\rho)=-\beta f_{0}^{\prime}(\rho)$, a relation that is equivalent to the compressibility equation of state. Utilizing this result and demanding that (8) be satisfied in the homogeneous limit leads to the requirement that the Fourier transform of $S(r ; \rho), S_{k}(\rho)$, be determined as the solution of

$$
c_{k}^{(2)}(\rho)=2 S_{k}(\rho)+\frac{\rho}{S_{0}^{\prime}(\rho)}\left[G_{k}^{\prime}(\rho)\right]^{2} .
$$

Taking the density derivative of (10) implies that the Fourier transform of the weight function $w_{k}(\rho)=g_{k}^{\prime}(\rho) / S_{0}^{\prime}(\rho)$ solves

$$
-\beta^{(1)}\left[c_{k}^{(2)}(\rho)\right]^{\prime}=2 f_{0}^{\prime \prime}(\rho) w_{k}(\rho)+\frac{d}{d \rho}\left[\rho f_{0}^{\prime \prime}(\rho) w_{k}^{2}(\rho)\right] .
$$

A closely related approach has been pursued by Curtin and Ashcroft ${ }^{4}$ (CA). What they do amounts to demanding that the second term on the right-hand side of (4) vanishes, so that

$$
\mathcal{F}_{\mathrm{ex}}\left[\rho, \rho_{0}\right]=\int d \mathbf{r} \rho(\mathbf{r}) f_{0}\left(\rho_{0}(\mathbf{r})\right) .
$$

In other words, $\rho_{0}(\mathbf{r})$ is determined from the requirement that the excess free-energy density be local in $\rho(\mathbf{r})$ and $\rho_{0}(\mathbf{r})$. Assuming (9), this implies that $\rho_{0}(\mathbf{r})$ is given by (7), with $w\left(\left|\mathbf{r}-\mathbf{r}^{\prime}\right| ; \rho_{0}(\mathbf{r})\right)=S\left(\left|\mathbf{r}-\mathbf{r}^{\prime}\right| ; \rho_{0}(\mathbf{r})\right) / S_{0}\left(\rho_{0}(\mathbf{r})\right) . \quad \mathcal{G}$ is then determined by requiring that $(8)$ be satisfied in the bulk homogeneous phase. The defining equation for $w_{k}(\rho)$ is easily found to be

$-\beta^{-1} c_{k}^{(2)}(\rho)=2 f_{0}^{\prime}(\rho) w_{k}(\rho)+\rho \frac{d}{d \rho}\left[f_{0}^{\prime}(\rho) w_{k}^{2}(\rho)\right]$.

Since by definition, $w_{k=0}(\rho)=1,(13)$ is consistent with the compressibility equation of state. Note the formal similarity between Eqs. (11) and (13).

\section{RESULTS AND DISCUSSION}

The equilibrium density distribution is that which minimizes the grand potential

$$
\Omega=\mathscr{F}[\rho]+\int d \mathbf{r}[V(\mathbf{r})-\mu],
$$

where $V(\mathbf{r})$ is an external potential and $\mu$ is the chemical potential of the fluid. In the following we consider a system of hard spheres of diameter $\sigma$ in contact with a hard wall described by the potential

$$
V(z)=\left\{\begin{array}{l}
\infty \text { for } z<-\sigma / 2 \\
0 \text { for } z \geq-\sigma / 2 .
\end{array}\right.
$$

All calculations are carried out using the Percus-Yevick compressibility equation of state and direct-correlation function $c^{(2)} .^{11}$ In order to compare with existing simulation data, ${ }^{12}$ profiles were calculated using the approximate Helmholtz free-energy functionals $\mathscr{F}[\rho]$ discussed in Sec. II for the bulk densities $\rho_{B} \sigma^{3}=0.575,0.813$, and 0.9135 . The results are shown in Figs. 1-3. The dashed lines are the predictions of the original MK theory; ${ }^{2}$ the results obtained using the GE (Ref. 3 ) and CA (Ref. 4) approximations are given by the solid lines. The difference between the predictions of these last two theories is too small to be resolved in the figures.

The essential features of these results can be summarized as follows. 
(i) Since the surface sum rule $\beta p=\rho\left(0^{+}\right)$is satisfied in all three approximations, they all yield a density at the wall which is consistent with the compressibility equation of state.

(ii) The "renormalized" theory of GE represents a substantial improvement over that of MK, implying that the requirement that the theory yields the correct $c^{(2)}(r)$ in the homogeneous bulk phase is of critical importance. In fact, the surprisingly close agreement between the predictions of the GE and CA WDA's indicates that this condition is the most important single element in determining the quality of the density profiles and, in particular, the phase of the density oscillations far from the wall.

Recently, Sokolowsky and Fischer ${ }^{13}$ have extended the approach of Ref. 2 to include three-particle direct correlations. This corresponds to going one order further in the expansion of (5) so that (6) is supplemented by the term

$$
\frac{1}{2} \int d \mathbf{r}^{\prime \prime} \int_{0}^{1} \lambda^{2} d \lambda c^{(3)}\left(\mathbf{r}, \mathbf{r}^{\prime}, \mathbf{r}^{\prime \prime} ; \lambda \rho_{0}(\mathbf{r})\right)\left[\rho\left(\mathbf{r}^{\prime \prime}\right)-\rho_{0}(\mathbf{r})\right] .
$$

To determine the three-particle direct-correlation function of the homogeneous fluid, they used the scheme recently suggested by Barrat, Hansen, and Pastore. ${ }^{14}$

The fact that this expansion is taken one order further than in Ref. 2 guarantees that (8) is satisfied in the homogeneous limit. However, an inconsistency appears at the next order, since the third functional derivative of $\mathcal{F}_{\mathrm{ex}}$ does not yield the correct three-point function. Nevertheless, their density profiles for a hard-sphere system at a hard wall represent a significant improvement over those of MK. In particular, the inclusion of the thirdorder term raises the maximum of the second peak and shifts it to somewhat smaller $z$ values; furthermore, the quality of the profiles near the first minimum also seems to improve. In spite of these improvements, the profiles predicted by the GE and CA WDA's discussed here are superior, particularly at higher densities and for $z / \sigma>1$.

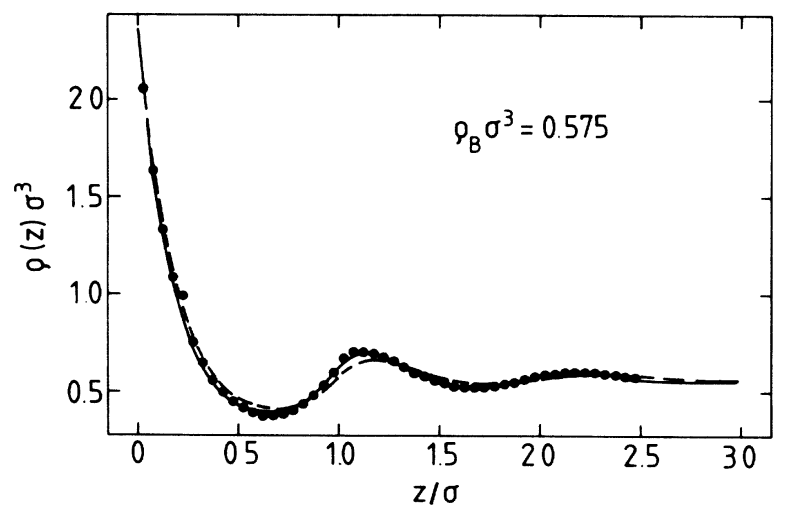

FIG. 1. Density profiles of a hard-sphere fluid at bulk density $\rho_{B} \sigma^{3}=0.575$ in contact with a hard wall. The dashed line is the prediction of the original Meister-Kroll theory (Ref. 2). The results obtained using the "renormalized" Groot-van der Eerden (Ref. 3) and Curtin-Ashcroft (Ref. 4) approximations are given by the solid line; the difference between the predictions of these two theories cannot be resolved in the figure. The points ( are Monte Carlo simulation results (Ref. 12).

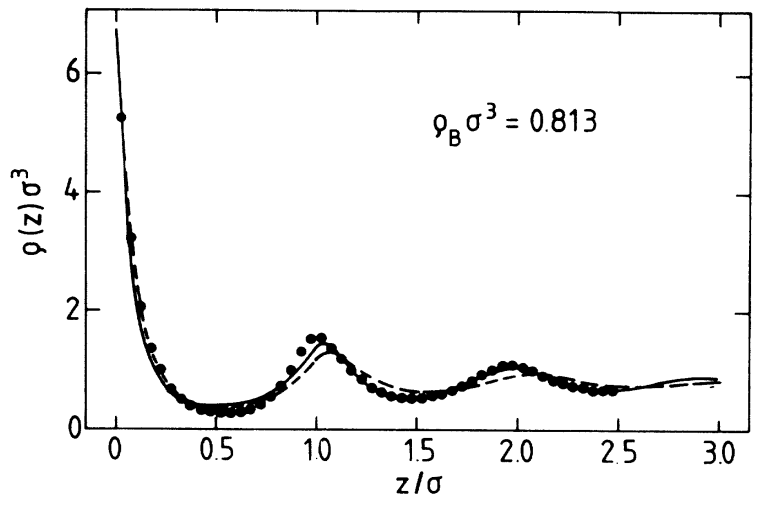

FIG. 2. Density profiles of a hard-sphere fluid at bulk density $\rho_{B} \sigma^{3}=0.813$ in contact with a hard wall. The notation is the same as in Fig. 1.

A major shortcoming of the weighted-densityfunctional theories of Refs. 3 and 4 is the relatively large amount of computational effort which is required. This feature does, in fact, restrict their usefulness. On the other hand, if one takes the weighted density to be independent of the spatial coordinate, these two approximations simplify considerably. Very recently, Denton and Ashcroft $^{15}$ have implemented this approach in the original CA WDA. The resulting theory retains many of the good features of the original weighted-density functional, but requires significantly less computational effort. In particularly, Denton and Ashcroft ${ }^{15}$ have shown that this simpler theory is about as accurate as the original CA WDA when applied to study the hard-sphere freezing transition.

It is straightforward to apply this same idea in the GE approximation. For fluids adsorbed at walls, it turns out that the resulting theory, as well as that of Denton and Ashcroft, are equivalent to the hypernetted-chain closure of the wall-particle Ornstein-Zernike equation. ${ }^{16}$ It is known that this closure yields density profiles that are in fairly good agreement with simulation data, provided $\rho_{B} \sigma \lesssim 0,4$. For higher densities this approximation overestimates the amplitude of the first few oscillations in

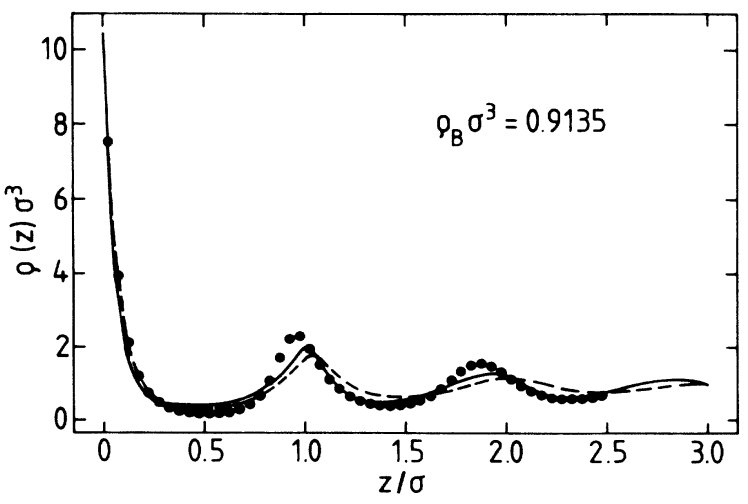

FIG. 3. Density profiles of a hard-sphere fluid at bulk density $\rho_{B} \sigma^{3}=0.9135$ in contact with a hard wall. The notation is the same as in Fig. 1. 
$\rho(z)$. Worse, the density at the wall no longer satisfies the surface sum rule $\beta p=\rho\left(0^{+}\right)$and, since the theory cannot describe two-phase (liquid-gas) coexistence, this approximation cannot describe wetting phenomena. It should be emphasized that these problems are not shared by the present set of theories which utilize spatially dependent weighted densities.

In closing, we have shown that the weighted-densityfunctional theories developed in Refs. 3 and 4 are of comparable accuracy when it comes to describing the structure of an inhomogeneous hard-sphere liquid. We expect that this is also the case for fluids with other pairinteraction potentials. The essential ingredient which both theories incorporate is the requirement that Eq. (8) be fulfilled in the homogeneous limit.

From this result, one might expect that both approximations are also of comparable accuracy when applied to study freezing of the hard-sphere liquid. This, however, is not the case. In particular, we have found that if the theory of Ref. 3 is reformulated for a spatially indepen- dent weighted density (as in the Denton-Ashcroft approximation), the equation for the weighted density [corresponding to Eq. (7)] has, in general, no solution in the solid phase; ${ }^{17}$ in contrast, the Denton-Ashcroft theory is known to yield rather good values for the hard-sphere freezing parameters. The reason for this problem can be traced to the position and density dependence of the first zero of the weight function $w_{k}(\rho)$. At present, we do not have a deeper understanding of why this theory, which does so well at describing liquid structure, fails so badly when applied to study freezing. In any case, approximations based on the ideas leading to approximation (12) appears to be superior in this respect.

\section{ACKNOWLEDGMENTS}

This material is based upon work supported by the North Atlantic Treaty Organization under a grant given to B.L.
${ }^{1}$ H. C. Anderson, D. Chandler, and J. D. Weeks, Adv. Chem. Phys. 34, 105 (1976).

${ }^{2}$ T. F. Meister and D. M. Kroll, Phys. Rev. A 31, 4055 (1985).

${ }^{3}$ R. D. Groot and J. P. van der Eerden, Phys. Rev. A 36, 4356 (1987).

${ }^{4}$ W. A. Curtin and N. W. Ashcroft, Phys. Rev. A 32, 2909 (1985).

${ }^{5}$ S. Nordholm, M. Johnson, and B. C. Freasier, Aust. J. Chem. 33, 2139 (1980).

${ }^{6}$ M. Johnson and S. Nordholm, J. Chem. Phys. 75, 1953 (1980).

${ }^{7}$ P. Tarazona, Mol. Phys. 52, 81 (1984).

${ }^{8}$ P. Tarazona, Phys. Rev. A 31, 2672 (1985).

${ }^{9}$ P. Tarazona and R. Evans, Mol. Phys. 52, 847 (1984).

${ }^{10}$ P. Tarazona, U. M. B. Marconi, and R. Evans, Mol. Phys. 60, 573 (1987).
${ }^{11}$ M. S. Wertheim, Phys. Rev. Lett. 10, 321 (1963); E. Thiele, J. Chem. Phys. 39, 474 (1963).

${ }^{12}$ R. D. Groot, N. M. Faber, and J. P. van der Eerden, Mol. Phys. 62, 861 (1987).

${ }^{13}$ S.Sokolowski and J. Fischer, Mol. Phys. 68, 647 (1989).

${ }^{14}$ J. L. Barrat, J. P. Hansen, and G. Pastore, Mol. Phys. 63, 747 (1988).

${ }^{15}$ A. R. Denton and N. W. Ashcroft, Phys. Rev. A 39, 4701 (1989).

${ }^{16}$ This has been shown for the Denton-Ashcroft theory by J. A. White and R. Evans [J. Phys.: Condens. Matter 2, 2435 (1990)].

${ }^{17} \mathrm{We}$ expect that this will also be the case for the original Meister-Kroll and Groot-van der Eerden theories. 